\title{
« The Shapley value as a guide to FRAND licensing agreements »
}

\author{
$\underline{\text { Auteurs }}$ \\ Pierre Dehez, Sophie Poukens \\ Document de Travail nº 2013 - 03
}

Mars 2013

Faculté des sciences économiques et de gestion

Pôle européen de gestion et d'économie (PEGE) 61 avenue de la Forêt Noire F-67085 Strasbourg Cedex

Secétariat du BETA Géraldine Manderscheidt Tél. : (33) 0368852069 Fax : (33) 0368852070 g.manderscheidt@unistra.fr www.beta-umr7522.fr 
The Shapley value as a guide to FRAND licensing agreements

\author{
by \\ Pierre Dehez \\ and \\ Sophie Poukens \\ CORE, University of Louvain \\ Louvain-la-Neuve, Belgium \\ email: pierre.dehez@uclouvain.be
}

February 2013

\begin{abstract}
We consider the problem of specifying Fair, Reasonable And Non-Discriminatory agreements faced by standard-setting organizations. Along with Layne-Farrar, Padilla and Schmalensee (2007), we model the problem as a cooperative game with transferable utility, allowing for patents to be weak in the sense that they have substitutes. Assuming that a value has been assigned to weak patents, we obtain a formula for the Shapley value that gives an insight into what FRAND agreements should look like.
\end{abstract}

Keywords: patent licensing, Shapley value, core

The authors are thankful to Paul Belleflamme and Pavitra Govindan for useful comments and suggestions on an earlier draft. 


\section{Introduction}

Over the last decades, standard setting organizations have played a decisive role in the development and commercialization of new technologies, and are still of growing importance. These collaborative bodies aim at promoting the emergence of a technical standard by ensuring the compatibility and interoperability of related devices. In order for a standard to effectively spread out as a reference, third parties must be guaranteed a straight access to the required intellectual property rights. This is why patents covering the different aspects of the standard are gathered together in a patent pool and licensed as a single package.

Augustin Cournot established in 1838 that a merger between two monopolists producing complementary goods generates both a higher joint profit and a lower final price by preventing the accumulation of margins. Shapiro (2001) points out that Cournot's complements problem arises when two or more firms own intellectual property rights required for the development of a product. If they do not coordinate, each patent holder will charge an excessive price in exchange of the access to its intellectual property right, without taking into account the impact of its decision on the level of sales of the final product and on the revenue of all patent holders.

The complements problem tends to be particularly important in the context of standard setting since access to all complementary intellectual property rights must be granted in order to ensure full compatibility with the standardized technology. This situation is referred to as the "tragedy of anti-commons" by Heller and Eisenberg (1998) because, in contrast with the tragedy of the commons, the fragmentation of the intellectual property rights leads to an underutilization of the associated standard.

Standard-setting organizations are groups of firms that set common standards for a particular technology to ensure compatibility and interoperability of the devices they manufacture. They have played a decisive role in the development and the commercialization of new technologies over the last decades and are still of growing importance. They are composed of upstream firms holding the intellectual property rights, downstream firms manufacturing goods based on the standard, and vertically integrated firms with characteristics of both categories. In order for a standard to effectively spread out as a reference, anyone must be guaranteed a straight access to the related technology. This is why patents covering the different aspects of the standard are gathered together in a patent pool and licensed as a single package. That prevents excessive independent pricing and reduces transaction costs through the possibility of one-stop shopping.

If there is no doubt about the global profitability of pooling complementary intellectual property rights, patent holders have to overcome substantial obstacles in order to ensure the 
pool formation: finding an agreement on the amount to be charged as a licensing fee and on the way to split the collected revenues among patent holders. In their case study of the MPEG-2 video patent pool, Lerner, Tirole and Strojwas (2003) illustrate the difficulty to reconcile heterogeneous objectives. ${ }^{1}$ One of the most debated issues during the pool formation was the licensing fee to be charged to licensees. Some members, like Columbia University, wanted to maximize the licensing revenue they receive, in contrast with other companies whose main purpose was to accelerate the adoption of the standard. This was the case of Sony, both licensor and licensee of MPEG-2 patents, who focuses on maximizing the sales of its standard-based products.

Many standard setting organizations, like for instance the $3 G$ Patent Platform Partnership or the RFID Consortium, require their members to stick to a particular commitment: the licensing of the intellectual property rights composing the standardized technology under Fair, Reasonable And Non-Discriminatory (FRAND) terms. This requirement aims mainly at avoiding abusive licensing terms that result from the monopoly power given to the pool members. Indeed, once a standard has become popular in an industry, it becomes very costly for a firm to produce a non-compliant good and patent owners are tempted to charge excessive licensing fees. This opportunistic behavior is referred to as the hold-up in the economic literature. Such conduct must be avoided since it is likely to have two adverse effects on economic efficiency: an increase in the deadweight loss resulting from market power and the selection of inferior technologies. According to Shapiro (2001), if standardsetting bodies like the International Telecommunications Unions (ITU), the European Telecommunications Standard Institute (ETSI) or the American National Standards Institute (ANSI) require their participants to accept to license any essential patent under FRAND terms before adopting a standard, this is primarily to face the risk of hold-up. The guarantee that no licensee could be subject to a discriminatory treatment and that the fee perceived from them stay in a reasonable interval strongly reduces the possibility of monopoly power abuses. It should be noted that, while FRAND predominates in Europe, it is usually restricted to RAND, i.e. Reasonable And Non-Discriminatory, in the United States. With the requirement for reasonableness and non-discrimination more oriented towards the hold-up problem, the American RAND commitment mainly focuses on ensuring economic efficiency issues, leaving aside fairness considerations.

Despite being often referred to, the notion of FRAND agreement suffers from a lack of definition. Several methods, beyond simple proportionality rules, have been advocated to give content to this ambiguous normative concept but none of them ever gained universal

\footnotetext{
${ }^{1}$ MPEG-2 is a digital video compression standard used in DVD and high-definition television.
} 
approval. Swanson and Baumol (2005) have suggested using the Efficient Component Pricing Rule (ECPR). This approach assumes that multiple technologies compete during the development phase and that the one winning an auction eventually becomes the effective standard. The auction implies that intellectual property right holders submit potential licensing fees to the downstream users who elect the winner. Swanson and Baumol show that the issue of the auction provides a benchmark for reasonable licensing fee as it reflects the state of competition before the adoption of the standard. They specify the non-discriminatory component by requiring licensing fees to be competitively neutral, i.e. such that patent holders are indifferent between licensing their technology to rivals and producing the final product themselves. For that purpose, they adapt the efficient component pricing rule in order for the license fee to compensate the licensor for the incremental licensing costs as well as for the opportunity cost of licensing the technology. Layne-Farrar, Padilla and Schmalensee (2007) instead have proposed to use the Shapley value, a sharing rule that compensates the members of a cooperative project on the basis of their marginal contributions. These two approaches rely on very different driving forces. The first one, based on market competition and efficient pricing, relies on non-cooperative principles while the second one is based on fairness principles, regardless of market conditions or efficiency. This led Layne-Farrar, Padilla and Schmalensee to consider the Swanson-Baumol method as a possible benchmark for RAND agreements in the United States, where economic efficiency is typically the foremost concern, and the Shapley value as a possible benchmark for FRAND agreements in the European Union, where fairness is viewed as important as efficiency.

Here we follow the proposal of Layne-Farrar, Padilla and Schmalensee to use cooperative game theory to analyze the compensation problem faced by standard-setting organizations. For this purpose, we translate the problem into a cooperative game with transferable utility (or side payments) and we consider two major solution concepts, namely the core and the Shapley value. The core identifies the set of socially stable allocations and the Shapley value provides an axiomatic method for a fair division. Our model differs however from theirs in that our "players" are the firms involved while their players are the ex-ante available patents. That means that we place ourselves after the formation of the pool while they place themselves at the outset of the pooling process, allowing firms with substitutable patents to compete. The immediate result is that any attempt to form a pool is due to fail because the core of the associated game retains only allocations that exclusively remunerate patents without competitor. The Shapley value instead defines an allocation at which substitutable patents are (equally) remunerated: there will always be a coalition of firms challenging such allocations. Our analysis instead assumes that the choice has been made regarding the firms that form the pool and that a value has been assigned to the patents that have substitutes. 
We construct a surplus sharing game, so called patent game, on the basis of the modeling framework introduced by Muto, Potters and Tijs (1989) who analyze the cooperative behavior of players willing to share a technology covered by a single patent. ${ }^{2}$ We extend their model to the case where the technology relies on multiple patents. We follow Layne-Farrar, Padilla and Schmalensee by allowing standards to be composed of two types of patents: those facing competition due to the existence of economically interchangeable alternatives and those being the unique contribution to a particular component of the standard. The latter are strong patents that are essential for the technology to stand up and cannot be invented around. The former are weak patents that, while being also essential, can be invented around at a known cost through some alternative solution. Firms owning strong patents have the ability (in essence equivalent to a veto right) to prevent the other players from realizing a profit, independently of the number of patents they own and of their relative importance. Firms detaining only weak patents do not have that blocking potential.

The identification of the strong patents is generally straightforward since in most technological standards, there are only few strong patents around which the standard is organized together with a (possibly large) number of weak patents. The assignment of a value to weak patents is the difficult part. Layne-Farrar, Padilla and Schmalensee (2007) recommend evaluating the worth of an essential patent on the basis of the number of competing alternatives it admits before the implementation of the standard. Linking the value of a patent to the availability of substitutable technologies relies on a specific valuation approach, known as option pricing that evaluates patents on the basis of their possible alternatives. If an alternative can easily be chosen, the incremental contribution of a patent is likely to be small. This finds an echo in Shapiro (2001) who states that if a patent can be easily invented around, "the patented technology contributes little if anything to the final product, and any reasonable royalty would be modest at best." It is clear that offering the same compensation to the owner of a weak patent and to the holder of a strong patent cannot be considered as fair. It is indeed quite intuitive that, if an essential patent admits technological alternatives, it can be considered as less valuable than other essential patents that do not face competing technologies. And if the cost of inventing around such a patent is small, so should be the compensation. In what follows, we assume that weak patents are well identified and that there is an agreement on their value.

In our patent game, firms are of different types. On the one hand, there is a fixed subset of patent owners who agree to license their intellectual property rights on FRAND terms. They

\footnotetext{
${ }^{2}$ They actually consider the problem of information sharing. Their analysis has been later extended by Potters and Tijs (1990) to the case where the information is owned by two players or more.
} 
are the members of the standard setting pool. They may be vertically integrated in the sense that they are not only active in the research field but also in the downstream product market. On the other hand, there are firms that want to be granted access to the whole set of essential patents in order to commercialize the standard-based product. We assume perfect patent protection, which implies that the only way to acquire the information covered by a patent is to be granted a license. Following Kamien and Tauman (1986), we distinguish patent licensing through a fixed fee and patent licensing through a royalty. The former is a fixed cost for giving access to the standard while the latter is a cost that is proportional to the quantity sold of the standard-based product. In what follow, we limit ourselves to the case of a fixed licensing fee.

The worth of a coalition of firms is defined on the basis of the profits it can secure on its own. We exclude strategic considerations by assuming that markets are partitioned: even if all firms can commercialize the same standard-based product, they operate on different markets. In other terms, there are no competition issues as licensors and licensees do not threaten each other's profit. We also exclude the exchange of information and knowledge that could take place within the patent pool. As a consequence, profits can simply be added. A coalition is in a position to generate profit only if it has been granted access to the whole set of required patents. It means that only coalitions that include all strong patent owners are able to generate a profit. That profit is simply the sum of the individual profits that its members can obtain by commercializing a standard-based product, from which the value of the missing weak patents has been removed.

These assumptions lead to a fairly simple formula using the axioms that underlie the Shapley value. It specifies the share of each participant in the total profit, from which one deduces who pays what and to whom. Beyond the numerical outcome, it suggests a number of properties that a FRAND agreement should have. Strong patent owners share equally an amount made of their total profit, augmented by the licensing fees they receive from the other firms and reduced by the licensing fees they pay to the weak patent owners. Hence, what a strong patent owner receives does not depend on the number of its strong patent nor on the value of the weak patent it possibly owns. Furthermore, non-profit organizations like universities or research institutions that own strong patents are entitled to the same share than any other strong patent owner. Weak patent owners receive licensing fees for an amount that is proportional to the value of the patents they own. This proportion is the same for all firms and depends only on the number of strong patent owners. It is at most one half and decreases with the number of strong patent owners. Weak patent owners as well as any other firm willing to use the standard have to give up an amount that is proportional to their profits. Again, the proportion depends only on the number of strong patent owners. It is at least one 
half and increases with the number of strong patent owners. Furthermore, the licensing fee paid by outside users only goes to the strong patent owners: outside users do not pay for the weak patents.

The remaining of the paper is organized as follows. Section 2 starts with generalities concerning cooperative games with transferable utility, followed by the definition of patent games and their properties. The core is defined in Section 3. It is shown that only a few inequalities are needed to characterize the core of a patent game. The Shapley value is defined in Section 4 and applied to patent games. Particular cases are analyzed and the Shapley value is compared to an alternative solution, namely the nucleolus. The last section offers some concluding remarks.

\section{Patent games}

\subsection{Games with transferable utility}

Cooperative games cover situations in which a group of individuals consider cooperating on a common project with the objective of maximizing collective welfare and allocating it between its members. It is assumed that utility is transferable through some commodity-money that allows for transfers (side-payments) among players. A cooperative game with transferable utility - TU-game - is defined by a characteristic function that captures the potential worth of each coalition of players. More specifically, given a set of players $N$, a characteristic function $v$ associates to each coalition $S \subset N$ a real number $v(S)$ that represents the minimum gain that coalition $S$ can realize without the participation of the others. By convention, $v(\varnothing)=0$. In particular, $v(i)$ is what player $i$ can obtain alone and $v(N)$ is the maximum amount that the "grand coalition" is able to generate. The object of the theory of cooperative game is the allocation of that maximum surplus between the players. It defines an agreement that specifies who pays what and who gets what. We denote by $X(N, v)$ the set of all allocations (also called "pre-imputations"):

$$
X(N, v)=\left\{x \in \mathbb{R}^{n} \mid \sum_{i \in N} x_{i}=v(N)\right\}
$$

The marginal contribution of player $i$ to coalition $S$ is defined by $v(S)-v(S \backslash i)$. Two players $i$ and $j$ are substitutable in a game $(N, v)$ if they contribute equally to all coalitions to which they belong: $v(S \backslash i)=v(S \backslash j)$ for all $S \subset N$ such that $i, j \in S$. A player $i$ is null in a game $(N, v)$ if he or she never contributes: $v(S)=v(S \backslash i)$ for all $S \subset N$.

A game $(N, v)$ is convex if for all subsets $S, T \subset N$

$$
v(S)+v(T) \leq v(S \cup T)+v(S \cap T) \text { for all } S \text { and } T \subset N
$$


Shapley (1971) has shown that a game is convex if and only if players' marginal contributions do not decrease with coalition size:

$$
i \in S \subset T \Rightarrow v(S)-v(S \backslash i) \leq v(T)-v(T \backslash i)
$$

As a consequence, the marginal contribution of a player is maximal at the grand coalition $N$. Convexity is a stronger property than superadditivity. A game $(N, v)$ is superadditive if the above inequalities holds only for disjoint subsets i.e. merging is not detrimental. It is essential if there is a potential gain in forming the grand coalition:

$$
\sum_{i \in N} v(i)<v(N)
$$

Notation: Lower-case letter will denote coalition sizes $(t=|T|, s=|S|, \ldots)$. Coalitions $\{i, j, k, \ldots\}$ will sometime be written as $i j k \ldots$

Imputations are individually rational allocations: no firm can individually object to an imputation. The set of imputations

$$
I(N, v)=\left\{x \in \mathbb{R}^{n} \mid \sum_{i \in N} x_{i}=v(N), x(i) \geq v(i) \text { for all } i \in N\right\}
$$

is a simplex. Superadditivity ensures that this set is non-empty. It reduces to the allocation $(v(1), \ldots, v(n))$ if the game is inessential.

Marginal contributions play a central role. Let $\Pi_{N}$ denote the set of all players' orderings. The marginal contributions vector is the imputation $\mu(\sigma)$ associated to the players' ordering $\sigma=\left(i_{1}, \ldots, i_{n}\right) \in \Pi_{N}$ is given by:

$$
\begin{aligned}
& \mu_{i_{1}}(\sigma)=v\left(i_{1}\right)-v(\varnothing)=v\left(i_{1}\right) \\
& \mu_{i_{k}}(\sigma)=v\left(i_{1}, \ldots, i_{k}\right)-v\left(i_{1}, \ldots, i_{k-1}\right) \quad(k=2, \ldots, n-1) \\
& \mu_{i_{n}}(\sigma)=v(N)-v\left(N \backslash i_{n}\right)
\end{aligned}
$$

There are $n$ ! marginal contribution vectors. They are not necessarily distinct. ${ }^{3}$

In what follows, we will refer to the unanimity game $\left(N, u_{T}\right)$ associated to a coalition $T \subset N$ :

$$
\begin{aligned}
u_{N}(S) & =1 & & \text { if } T \subset S \\
& =0 & & \text { if } T \not \subset S
\end{aligned}
$$

For any given $N$, the collection of unanimity games $\left(u_{T} \mid T \subset N, T \neq \varnothing\right)$ forms a basis of the vector space of set functions on $N$.

\footnotetext{
${ }^{3}$ Except for strictly convex games (Shapley, 1971).
} 


\subsection{Patent games}

A patent situation is defined by a set $N=\{1, \ldots, n\}$ of $n$ firms, the subset $M \subset N$ of $m$ firms owning strong patents, the values of the weak patents owned by each firm, $p_{i} \geq 0$ for firm $i$, and the additional net sales (profit) that each firm plans to obtain from operating the standard, $\pi_{i} \geq 0$ for firm $i$. We assume that all patents have been recognized to be essential. That means that there are four types of firms: firms owning strong patents and possibly also weak patents ( $\left.i \in M, p_{i} \geq 0\right)$, firms owning only weak patents $\left(i \notin M, p_{i}>0\right)$ and firms owning no patent at all $\left(i \notin M, p_{i}=0\right)$. The latest are those who are willing to use the standard covered by the patent pool formed by the other firms. As we allow for $\pi_{i}=0$, there is room for non-profit organization like universities or research institutions to be included in the set of patent owners. We assume that there is at least one firm owning strong patents: $m \geq 1$. The case where there is no strong patent will be considered separately.

The patent game $(N, v)$ is the TU-game associated to the patent situation $(N, M, p, \pi)$ and defined by the characteristic function:

$$
\begin{aligned}
v(S) & =\sum_{i \in S} \pi_{i}-\sum_{i \notin S} p_{i} & & \text { if } M \subset S \\
& =0 & & \text { if } M \not \subset S
\end{aligned}
$$

Here $v(S)$ is the total profit that coalition $S$ can ensure itself if it forms, net of the value of the weak patents needed to meet the standard. The implicit assumption is that firms operate on separate markets so that profits can simply be added. In particular $v(N)$ is the total profit $\sum_{i \in N} \pi_{i}$. It is that amount that has to be divided among the $n$ firms.

We observe that the value of the weak patents held by strong patent owners do not enter in the definition of the patent game: as far as patents are concerned, the only data actually needed are the identity of the firms owning strong patents and the value of the patents owned by firms without strong patent. Furthermore, $v(i)=0$ for all $i$ whenever $m \geq 2$.

To ensure that $v(S) \geq 0$ for all $S \subset N$, we introduce the following additional assumption:

$$
\sum_{i \in M} \pi_{i} \geq \sum_{i \in N \backslash M} p_{i}
$$

It says that the coalition formed by the strong patent owners can generate enough profit to cover the cost of all missing weak patents.

Marginal contributions of a player $i$ to coalitions containing $M$ are given by:

$$
\begin{aligned}
v(S)-v(S \backslash i) & =v(S) \quad \text { if } i \in M \\
& =\pi_{i}+p_{i} \quad \text { if } i \notin M
\end{aligned}
$$


Otherwise, they are equal to zero. Hence, firms owning strong patents are substitutable players. There is indeed no difference between strong patent owners: each of them has a veto right, independently of the number and relative importance of the patents it owns.

Consider the following patent situation involving four firms:

firm 1 owns only strong patents $\left(p_{1}=0\right)$

firm 2 owns strong patents and weak patents for a value $p_{2}>0$

firm 3 owns only weak patents for a value $p_{3}>0$

firm 4 is an outside user $\left(p_{4}=0\right)$

Here $M=\{1,2\}$ and the "winning" coalitions are those involving the first two firms:

$$
\begin{aligned}
& v(12)=\pi_{1}+\pi_{2}-p_{3} \\
& v(123)=\pi_{1}+\pi_{2}+\pi_{3} \\
& v(124)=\pi_{1}+\pi_{2}+\pi_{4}-p_{3} \\
& v(1234)=\pi_{1}+\pi_{2}+\pi_{3}+\pi_{4}
\end{aligned}
$$

Lemma Patent games are essential and convex (and thereby superadditive).

Proof Essentiality is immediate. To check convexity, let $S$ and $T$ be any two subsets of $N$. We define $p_{0}=\sum_{i \in N} p_{i}$ and consider the three possible cases. Using (3) and the nonnegativity of $v(S)$ guaranteed by (4), we have successively:

(i) $M \subset S$ and $M \not \subset T$

$$
\begin{aligned}
& v(S \cap T)=v(T)=0 \\
& v(S)=\sum_{i \in S} \pi_{i}+\sum_{i \in S} p_{i}-p_{0} \\
& v(S \cup T)=\sum_{i \in S} \pi_{i}+\sum_{i \in S} p_{i}+\sum_{i \in T} \pi_{i}+\sum_{i \in T} p_{i}-\sum_{i \in S \cap T} \pi_{i}-\sum_{i \in S \cap T} p_{i}-p_{0} \\
& =\sum_{i \in S} \pi_{i}+\sum_{i \in S} p_{i}+\sum_{i \in T \backslash S} \pi_{i}+\sum_{i \in T \backslash S} p_{i}-p_{0} \geq \sum_{i \in S} \pi_{i}+\sum_{i \in S} p_{i}-p_{0}
\end{aligned}
$$

(ii) $M \not \subset S$ and $M \not \subset T$

$$
\begin{aligned}
& v(S)=v(T)=v(S \cap T)=0 \\
& v(S \cup T) \geq 0
\end{aligned}
$$

(iii) $M \subset S \cap T$

$$
v(S)+v(T)=v(S \cup T)+v(S \cap T)=\sum_{i \in S} \pi_{i}+\sum_{i \in S} p_{i}+\sum_{i \in T} \pi_{i}+\sum_{i \in T} p_{i}-2 p_{0}
$$

Hence, the inequality (1) holds in all three cases, confirming convexity. 


\section{The core}

\subsection{General definition}

The core of a TU-game $(N, v)$ is the set of allocations that no coalition can improve upon:

$$
C(N, v)=\left\{x \in \mathbb{R}^{n} \mid \sum_{i \in N} x_{i}=v(N), \sum_{i \in S} x_{i} \geq v(S) \text { for all } S \subset N\right\}
$$

Alternatively, no coalition is in a position to formulate an objection against a core allocation. Geometrically, the core is a polytope whose dimension is at most $n-1$. It may be empty. In the case of a convex game the core is the non-empty polytope whose vertices are the marginal contribution vectors. Furthermore, there is no ambiguity: two convex games coincide if and only if they have the same core (Shapley, 1971).

\subsection{The core of a patent game}

The core of a patent game is actually defined by at most $2 n-m$ inequalities. Beyond nonnegativity, an allocation is in the core of a patent game if the firms without strong patents do not get more than their stand-alone profit augmented by the value of the weak patents they own.

Proposition 1 The core of the patent game defined by $(N, M, p, \pi)$ is given by:

$$
C(N, M, p, \pi)=\left\{x \in \mathbb{R}_{+}^{n} \mid \sum_{i \in N} x_{i}=\sum_{i \in N} \pi_{i}, x_{i} \leq \pi_{i}+p_{i} \text { for all } i \in N \backslash M\right\}
$$

Proof We will proceed in two steps. Let's denote by $(N, v)$ the TU-game associated to the patent situation $(N, M, p, \pi)$. It is easily verified that any core allocation $x$ satisfies the following equivalent inequalities:

$$
\sum_{i \in S} x_{i} \leq v(N)-v(N \backslash S) \text { for all } S \subset N
$$

i.e. no coalition can obtain more than its contribution to the grand coalition. Applied to an individual player $i$, it reads

$$
x_{i} \leq v(N)-v(N \backslash i)
$$

The first half of the proposition then follows from the fact that $v(N)-v(N \backslash i)=\pi_{i}+p_{i}$ for all $i \notin M$.

Consider now an allocation $x \in \mathbb{R}_{+}^{n}$ satisfying $x_{i} \leq \pi_{i}+p_{i}$ for all $i \notin M$. If $M \not \subset S$, we have:

$$
v(S)=0 \leq \sum_{i \in S} x_{i}
$$


If instead $M \subset S$, we have:

$$
\sum_{i \in S} x_{i}=\sum_{i \in N} \pi_{i}-\sum_{i \notin S} x_{i} \geq \sum_{i \in N} \pi_{i}-\sum_{i \notin S}\left(\pi_{i}+p_{i}\right)=\sum_{i \in S} \pi_{i}-\sum_{i \notin S} p_{i}=v(S)
$$

Hence $x \in C(N, v)$. This completes the proof of the equivalence.

Two opposite forces are present in the definition of the core of a patent game. On the one hand, the core contains the allocations that give the total value of the game to one of the strong patent owner: for given any $j \in M$, the allocation $x$ satisfying $x_{i}=\sum_{i \in N} \pi_{i}$ and $x_{i}=0$ for all $i \neq j$ belongs to the core. The core considers all strong patents as equivalently indispensable: if one strong patent owner withdraws from the pool, no profit is obtained at all. Every strong patent owner has a veto power and can claim the entire value of the game since its marginal contribution to the grand coalition is the whole cooperative surplus. On the other hand, each intellectual property right owner may end up with nothing since the dissemination of essential patents across multiple owners implies that no one is able to realize a profit by standing alone. All patent holders are competing with each other to capture the greatest possible share of the cooperative surplus. While being a cooperative concept, the core highlights the competitive forces that may exist within the game.

Consider the case of three firms where firm 1 is alone to own strong patents, firm 2 owns weak patents and firm 3 is an outside user. The associated characteristic function is given by:

$$
\begin{aligned}
& v(1)=\pi_{1}-p_{2} \\
& v(12)=\pi_{1}+\pi_{2} \\
& v(13)=\pi_{1}+\pi_{3}-p_{2} \\
& v(123)=\pi_{1}+\pi_{2}+\pi_{3}
\end{aligned}
$$

As Figure 1 confirms, the only active inequalities in the definition of the core are:

$$
\begin{aligned}
& 0 \leq x_{2} \leq \pi_{2}+p_{2} \\
& 0 \leq x_{3} \leq \pi_{3}
\end{aligned}
$$

while the inequalities $\pi_{1}-p_{2} \leq x_{1} \leq \pi_{1}+\pi_{2}+\pi_{3}$ are redundant.

The case of 3-firm in which $M=\{1,2\}$, the characteristic function is defined by:

$$
\begin{aligned}
& v(12)=\pi_{1}+\pi_{2}-p_{3} \\
& v(123)=\pi_{1}+\pi_{2}+\pi_{3}
\end{aligned}
$$

We know from (5) that the only active inequalities in the definition of the core are:

$$
0 \leq x_{3} \leq \pi_{3}+p_{3}
$$




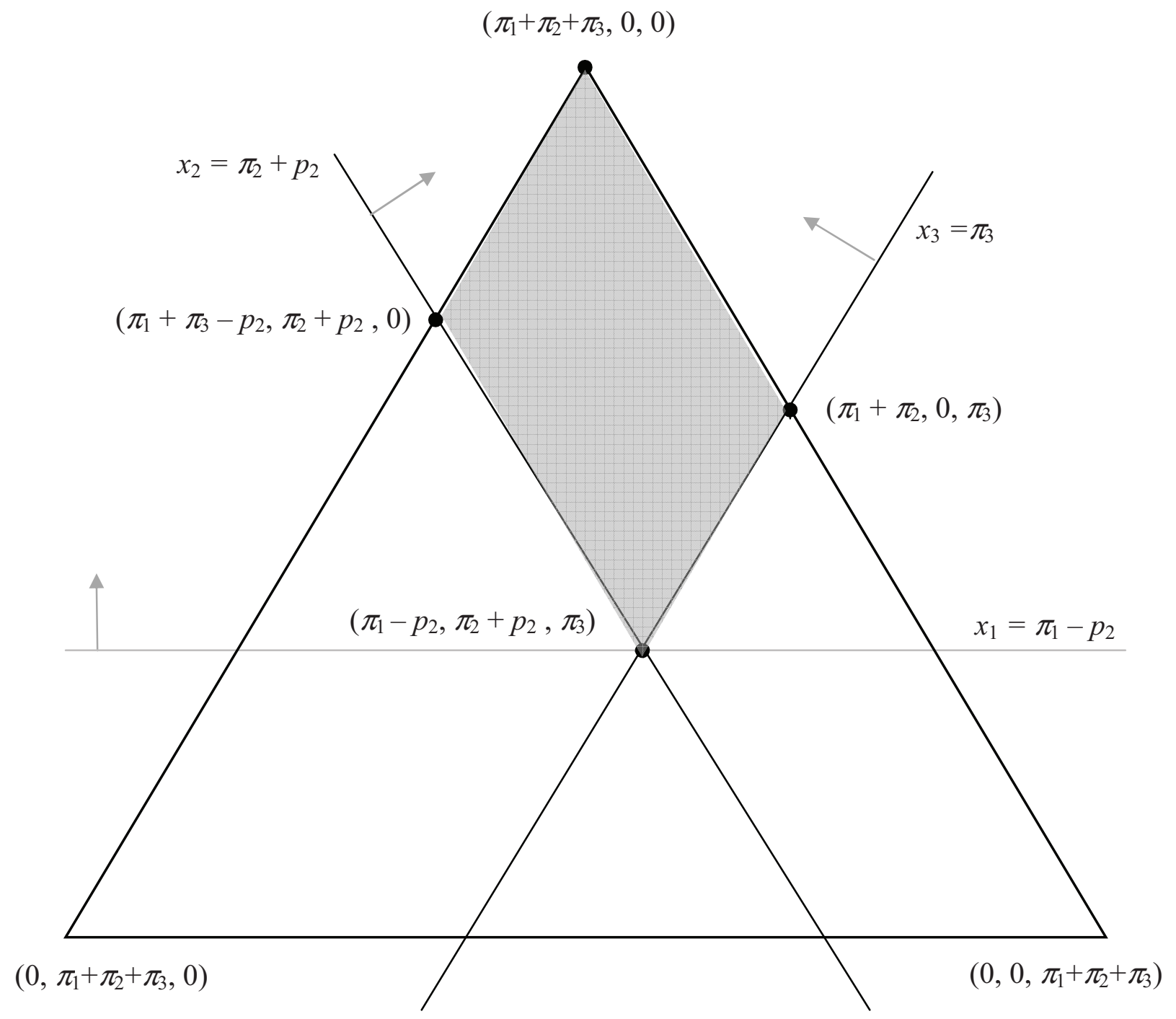

Figure 1 


\section{Shapley value}

\subsection{General definition}

The Shapley value is an allocation rule based on marginal contributions. It can be defined as the average marginal contribution vector, considering all permutations:

$$
S V_{i}(N, V)=\frac{1}{n !} \sum_{\sigma \in \Pi_{N}} \mu_{i}(\sigma) \quad i=1, \ldots, n
$$

Shapley (1953) has shown that it is the only efficient and additive allocation rule that is symmetric and satisfies the null player property. Efficiency simply requires that the value of the game be exactly allocated. Additivity is an independence property that guarantees that, when confronted to several games, a player evaluates them independently of each other. It is this property gives to the Shapley value its linear structure. Symmetry and the null player property require that substitutable players are treated equally and null players get zero.

There are alternative axiomatizations. Young (1985) has shown that the Shapley value is the only efficient, symmetric and marginalist allocation rule in the sense that what a player receives exclusively depends on the value of his or her marginal contributions.

Applied to a convex game, the Shapley value (6) defines a core allocation because core's vertices are precisely the marginal contribution vectors.

The Shapley value can equivalently be defined as the expected marginal contribution to a coalition chosen at random, given that coalitions of same size are equally likely and coalitions' size have all the same probability $1 / n$ to occur. This gives rise to the following formulation:

$$
S V_{i}(N, v)=\sum_{\substack{S \subset N \\ i \in S}} \alpha_{n}(s)(v(S)-v(S \backslash i)) \quad i=1, \ldots, n
$$

where the weights depend only on coalitions' size: ${ }^{4}$

$$
\alpha_{n}(s)=\frac{(s-1) !(n-s) !}{n !} \text {. }
$$

\subsection{The Shapley value of a patent game}

The following proposition establishes the formula that result from the definition of the Shapley value.

\footnotetext{
${ }^{4}$ The product of $\alpha_{n}(s)$ by the number of coalitions of size $s$ containing a given player is indeed equal to $1 / n$.
} 
Proposition 2 The Shapley value of the patent game defined by $(N, M, p, \pi)$ is given by:

$$
\begin{aligned}
& \text { for } i \notin M: S V_{i}(N, M, p, \pi)=\frac{1}{m+1}\left(\pi_{i}+p_{i}\right) \\
& \text { for } i \in M: S V_{i}(N, M, p, \pi)=\frac{1}{m} \sum_{j \in M} \pi_{j}+\frac{1}{m+1} \sum_{j \notin M} \pi_{j}-\frac{1}{m(m+1)} \sum_{j \notin M} p_{j}
\end{aligned}
$$

Proof Let us define $\theta_{i}=\pi_{i}+p_{i}$ and $p_{0}=p(N)$. Using (3), $v$ can be decomposed as $v=v_{1}-v_{2}$ where the games $\left(N, v_{1}\right)$ and $\left(N, v_{2}\right)$ are defined by:

$$
\begin{aligned}
v_{1}(S) & =\sum_{i \in S} \theta_{i} & & \text { if } M \subset S \\
& =0 & & \text { if not }
\end{aligned}
$$

and

$$
\begin{aligned}
v_{2}(S) & =p_{0} & & \text { if } M \subset S \\
& =0 & & \text { if not }
\end{aligned}
$$

To compute the Shapley value of the game $\left(N, v_{1}\right)$, we only have to compute what any player outside $M$ receives. Looking at players' orderings, we observe that the marginal contribution of a player $i \notin M$ is either 0 or $\theta_{i}$. It is $\theta_{i}$ if and only if player $i$ is preceded by all players in $M$. For a given ordering of the players in $M \cup\{i\}$ with player $i$ last, there are $C_{n}^{m+1}$ ways to place them. Hence, the number of times $i$ is preceded by the players in $M$ is given by:

$$
C_{n}^{m+1} m !(n-m-1) !=\frac{n !}{(m+1) !(n-m-1) !} m !(n-m-1) !=\frac{n !}{m+1}
$$

Using the first formulation (6), we get:

$$
S V_{i}\left(N, v_{1}\right)=\frac{1}{m+1} \theta_{i} \quad \text { for all } i \notin M
$$

Combining symmetry and efficiency, we obtain:

$$
S V_{i}\left(N, v_{1}\right)=\frac{1}{m} \sum_{i \in M} \theta_{i}+\frac{1}{m+1} \sum_{i \notin M} \theta_{i} \quad \text { for all } i \in M
$$

The game $\left(N, v_{2}\right)$ is the unanimity game $\left(N, p_{0} u_{M}\right)$ associated to the set $M$. Its Shapley value is given by:

$$
\begin{aligned}
S V_{i}\left(N, v_{2}\right) & =0 & & \text { for all } i \notin M \\
& =\frac{p_{0}}{m} & & \text { for all } i \in M
\end{aligned}
$$


Indeed players outside $M$ are dummies while the players in $M$ are substitutable. By linearity of the Shapley value, $S V(N, v)=S V\left(N, v_{1}\right)-S V\left(N, v_{2}\right)$ resulting in (8).

According to (8), strong patent owners receive together licensing fees from the other firms for an amount that is proportional to their profits:

$$
\frac{m}{m+1} \sum_{i \notin M} \pi_{i}
$$

Strong patent owners pay together to the weak patent owners a compensation that is proportional to the total value of the weak patents:

$$
\frac{1}{m+1} \sum_{i \notin M} p_{i}
$$

These two amounts are distributed equally among the strong patent owners who also share equally their profit. By their nature, the number of strong patents firms own and their relative importance plays no role: the Shapley value treats them equally. Furthermore, they get no compensation for the weak patents they own. Any other firm $i \notin M$ pays a part of its profit as licensing fee for using the strong patents equal to

$$
\frac{m}{m+1} \pi_{i}
$$

and, if it owns weak patents, it receives licensing fees from strong patent owners equal to

$$
\frac{1}{m+1} p_{i}
$$

Hence, the strong patent owners collect a large proportion of the profit generated by weak patent owners and outside users, a proportion that is greater than $1 / 2$ and increases with the number of strong patent owners. For $m$ large, they actually collect most of their profit.

As a consequence, if firms know that the Shapley will be used as a compensation scheme, individual firms have a strong incentive to submit strong patents. Actually, it is enough for a firm to convince its partners that it owns one strong patent that is essential for the standard, whatever are the other patents it possibly owns.

The situation of non-profit organizations depends on the kind of patent they own. If they own strong patents, they get the same income as any other strong patent owner. If they only own weak patents, they get licensing fees proportional to the value of their patents, exactly like any other weak patent owner. 
We could have started with the game restricted to the members of the patent pool, a situation where either $i \in M$ and/or $p_{i}>0$ for all $i$. Indeed, outside users do not pay for weak patents. They pay a licensing fee to the strong patent owners only and that fee is proportional to their profits. Furthermore, that proportion is the same than the proportion that applies to weak patent owners i.e. $m /(m+1)$. We observe that weak patent owners and outside users are treated in the same way: the strong patent owners reap at least one half of the profits they collect.

By convexity, the Shapley value belongs to the core. It recommends a system of licensing fees that is stable: neither individual firms nor coalitions of firms have an interest in challenging it.

In the 4-firm example, there are 8 distinct marginal contribution vectors. They are given by the following table together with their multiplicity:

\begin{tabular}{|c|c|c|c|c|}
\hline 0 & $\pi_{1}+\pi_{2}-p_{3}$ & $\pi_{3}+p_{3}$ & $\pi_{4}$ & 2 \\
\hline 0 & $\pi_{1}+\pi_{2}+\pi_{3}$ & 0 & $\pi_{4}$ & 2 \\
\hline 0 & $\pi_{1}+\pi_{2}+\pi_{3}+\pi_{4}$ & 0 & 0 & 6 \\
\hline 0 & $\pi_{1}+\pi_{2}+\pi_{4}-p_{3}$ & $\pi_{3}+p_{3}$ & 0 & 2 \\
\hline$\pi_{1}+\pi_{2}-p_{3}$ & 0 & $\pi_{3}+p_{3}$ & $\pi_{4}$ & 2 \\
\hline$\pi_{1}+\pi_{2}+\pi_{3}$ & 0 & 0 & $\pi_{4}$ & 2 \\
\hline$\pi_{1}+\pi_{2}+\pi_{3}+\pi_{4}$ & 0 & 0 & 0 & 6 \\
\hline$\pi_{1}+\pi_{2}+\pi_{4}-p_{3}$ & 0 & $\pi_{3}+p_{3}$ & 0 & 2 \\
\hline
\end{tabular}

The corresponding Shapley value is given by:

$$
\begin{aligned}
& S V_{1}(N, M, p, \pi)=S V_{2}(N, M, p, \pi)=\frac{\pi_{1}+\pi_{2}}{2}+\frac{\pi_{3}+\pi_{4}}{3}-\frac{p_{3}}{6} \\
& S V_{3}(N, M, p, \pi)=\frac{\pi_{3}+p_{3}}{3} \\
& S V_{4}(N, M, p, \pi)=\frac{\pi_{4}}{3}
\end{aligned}
$$

It is interesting to compare the Shapley value to the simple average of the marginal contribution vectors that is obtained without taking their multiplicity into account. In general, the core of a patent game has $2^{n-m-1}$ vertices, each corresponding to a given marginal contribution vector. Among them, $2^{n-m-2}$ give an amount $\pi_{i}+p_{i}$ to any given firm $i \notin M$ while all others give 0 . As a consequence, the average of core's vertices imposes to firms outside $M$ to give up half of their profit, independently of the number of strong patent 
owners. Weak patent owners receive from strong patent owners half of the value of their patents as compensation. As far as strong patent owners are concerned, they share an equal amount. In the framework of the 4-firm example, it gives the following allocation:

$$
\begin{aligned}
& A V_{1}(N, M, p, \pi)=A V_{2}(N, M, p, \pi)=\frac{\pi_{1}+\pi_{2}}{2}+\frac{\pi_{3}+\pi_{4}}{4}-\frac{p_{3}}{4} \\
& A V_{3}(N, M, p, \pi)=\frac{\pi_{3}+p_{3}}{2} \\
& A V_{4}(N, M, p, \pi)=\frac{\pi_{4}}{2}
\end{aligned}
$$

\subsection{The Shapley value in particular cases}

No weak patent $(p=0)$. The Shapley value then reduces to:

$$
\begin{aligned}
& \text { for } i \notin M: \quad S V_{i}(N, M, \pi)=\frac{1}{m+1} \pi_{i} \\
& \text { for } i \in M: \quad S V_{i}(N, M, \pi)=\frac{1}{m} \sum_{j \in M} \pi_{j}+\frac{1}{m+1} \sum_{j \notin M} \pi_{j}
\end{aligned}
$$

Every firm owns strong patents $(m=n)$. In that case, the Shapley value is the equal division:

$$
S V_{i}(N, M, \pi)=\frac{1}{n} \sum_{i \in N} \pi_{i} \text { for all } i \in N
$$

and it is actually the only conceivable allocation. The associated patent game is indeed the unanimity game on $N$ defined by:

$$
\begin{aligned}
u_{N}(S) & =\pi(N) & & \text { if } S=N \\
& =0 & & \text { if } S \neq N
\end{aligned}
$$

Only one player holds strong patents $(m=1){ }^{5}$ In that case, the Shapley value coincides with the average of core's vertices: the strong firm (say firm 1) receives half of the net profits of the other firms and returns them half of the value of the weak patents they own:

$$
S V_{1}(N, M, p, \pi)=\pi_{1}+\frac{1}{2} \sum_{i \in N \backslash 1} \pi_{i}-\frac{1}{2} \sum_{i \in N \backslash 1} p_{i}
$$

\footnotetext{
${ }^{5}$ This case corresponds to the monopolistic information game studied by Muto et al. (1989) with $p=0$.
} 
The amount allocated to the weak patent owners is given by:

$$
S V_{i}(N, M, p, \pi)=\frac{1}{2}\left(\pi_{i}+p_{i}\right) \text { for all } i \neq 1
$$

The patent owner collects half of the profit obtained by the other firms and compensates the firm owning weak patents: all firms except the strong patent owner keep half of their profit augmented by the value of their weak patent. In the case of three firms, the Shapley value is given by the following allocation:

$$
\left(\pi_{1}+\frac{\pi_{2}-p_{2}}{2}+\frac{\pi_{3}}{2}, \frac{\pi_{2}+p_{2}}{2}, \frac{\pi_{3}}{2}\right)
$$

Only weak patents $(m=0)$. That situation is not covered by the preceding analysis. The patent game can be written as:

$$
v(S)=\sum_{i \in S} \pi_{i}-\sum_{i \notin S} p_{i}=\pi(S)+p(S)-p_{0} \text { for all } S \subset N
$$

In the absence of strong patents, it is always possible for a coalition to realize profit since all missing patents can be bypassed as long as the required investment is made. This is an additive characteristic function up to a constant. By additivity, the Shapley value is simply given by: ${ }^{6}$

$$
S V_{i}(N, p, \pi)=\pi_{i}+p_{i}-\frac{p_{0}}{n} \text { for all } i \in N
$$

It recommends that each firm keeps the profit that it can realize by commercializing the standard-based product. All players uniformly support the value of the whole patent set and the value of each patent is completely redistributed to the firm holding it. Therefore, a patent holder is compensated in proportion to the value of the patents in its possession and effectively perceives a compensation if the value of his patents is superior to the per capita value of the whole patent set.

\subsection{Alternative solution: the nucleolus}

The theory of cooperative games has produced many solution concepts. However, in the framework of convex games, they tend to converge: the core is the unique stable set (in the sense of von Neumann and Morgenstern) and it coincides with the bargaining set (with respect to the grand coalition); the kernel and the nucleolus coincide; the Shapley value like the nucleolus is centrally located in the core. ${ }^{7}$

\footnotetext{
${ }^{6}$ It corresponds to a particular case of the compensation problem studied by Dehez and Tellone (2013) in a data sharing context.

${ }^{7}$ See Shubik (1982) for an overview of these concepts.
} 
As compared to the nucleolus, the Shapley value has some clear advantages: it is based on marginal contributions and, in the context of patent games, it gives rise to a fairly simple formula with clear-cut recommendations.

The nucleolus was introduced by Schmeidler (1969). It can be viewed as a refinement of the least core, a concept introduced later by Maschler, Peleg and Shapley (1979). Both concepts are concerned with the minimization of coalitions' dissatisfaction as measured by the difference between what they are worth and what they actually get. The least core is a set and the nucleolus is an element of the least core. More specifically, the loss for a coalition $S$ associated to an allocation $x$ is measured by $e(x, S)=v(S)-x(S)$. The least core is the set of allocations that minimize the largest loss:

$$
\operatorname{Min}_{x \in X(N, v)} \operatorname{Max}_{\substack{S \subset N \\ S \neq \varnothing, N}} e(x, S)
$$

It is a nonempty set whose dimension is at most $n-2$ and it is obviously a subset of the core if the latter is nonempty. The nucleolus goes further by comparing losses lexicographically, so as to eventually retain a unique allocation.

To quote Maschler, Peleg and Shapley (1979), the nucleolus is "the result of an arbitrator's desire to minimize the dissatisfaction of the most dissatisfied coalition." When the core is nonempty, it is more appropriate to reverse this quotation by saying that the nucleolus is "the result of an arbitrator's desire to maximize the satisfaction of the least satisfied coalition." As a rule, the nucleolus shares all but one of the original axioms underlying the Shapley value: it is symmetric and satisfies the null player property but it does not satisfy additivity. As a consequence, it is not a linear rule and it does not satisfy Young's marginalism axiom.

Computing the nucleolus is in general not straightforward and, applied to patent games, it does not result into a simple formula except in particular cases. If there is a single strong patent owner, the nucleolus and the Shapley value happen to coincide. ${ }^{8}$ In the case where there is no strong patent, the core is a simplex and therefore all "core center" solutions coincide.

To illustrate what happens outside these particular cases, let us consider the 3-firm example in which $M=\{1,2\}$. The associated characteristic function is defined by $v(12)=\pi_{1}+\pi_{2}-p_{3}$ and $v(123)=\pi_{1}+\pi_{2}+\pi_{3}$. The nucleolus takes two forms, either the equal division allocation or the average of core's vertices, depending on the parameters, in particular on the value of $p_{3}$.

\footnotetext{
${ }^{8}$ This is proven in Muto, Potters and Tijs (1989),
} 
Computing the nucleolus is easy because the least-core turns out to be a singleton. Knowing that the nucleolus satisfies the axiom of symmetry, it must be of the form

$$
\left(\frac{\pi_{1}+\pi_{2}+\pi_{3}-a}{2}, \frac{\pi_{1}+\pi_{2}+\pi_{3}-a}{2}, a\right)
$$

where $0 \leq a \leq \pi_{1}+\pi_{2}+\pi_{3}$. The losses can then be written in terms of the single parameter $a$ :

$$
\begin{aligned}
& e(a, 1)=e(a, 2)=\frac{a}{2}-\frac{\pi_{1}+\pi_{2}+\pi_{3}}{2} \\
& e(a, 3)=-a \\
& e(a, 12)=a-\left(\pi_{3}+p_{3}\right) \\
& e(a, 13)=e(a 23)=-\frac{a}{2}-\frac{\pi_{1}+\pi_{2}+\pi_{3}}{2}
\end{aligned}
$$

where $e(a, 13)$ and $e(a, 23)$ can be disregarded because they always fall below $e(a, 1)$. The dotted line in Figurer 2 is the graph of $e(a, 12)$ for $p_{3}$ equal to a critical value $\bar{p}_{3}$ given by:

$$
\bar{p}_{3}=\frac{2}{3}\left(\pi_{1}+\pi_{2}\right)-\frac{1}{3} \pi_{3}
$$

Taking (4) into account, the solution is given either by the intersection between $e(a, 1)$ and $e(a, 3)$ if $0 \leq p_{3} \leq \bar{p}_{3}$ or by the intersection between $e(a, 12)$ and $e(a, 3)$ if $\bar{p}_{3}<p_{3} \leq \pi_{1}+\pi_{2}$ :

$$
\begin{aligned}
a & =\frac{\pi_{3}+p_{3}}{2} \quad \text { if } 0 \leq p_{3} \leq \bar{p}_{3} \\
& =\frac{\pi_{1}+\pi_{2}+\pi_{3}}{3} \text { if } \bar{p}_{3}<p_{3} \leq \pi_{1}+\pi_{2}
\end{aligned}
$$

It is the equal division in the second case: the total profit is equally distributed among the three firms. In the first case, it is the average of core's vertices:

$$
\begin{aligned}
& A V_{1}(N, M, \pi, p)=A V_{2}(N, M, \pi, p)=\frac{\pi_{1}+\pi_{2}}{2}+\frac{\pi_{3}-p_{3}}{4} \\
& A V_{3}(N, M, \pi, p)=\frac{\pi_{3}+p_{3}}{2}
\end{aligned}
$$

In both cases, the nucleolus differs from the Shapley value that is defined by:

$$
a=\frac{\pi_{3}+p_{3}}{3}
$$

independently of the value of $p_{3} \leq \pi_{1}+\pi_{2}$. 
We observe that $\bar{p}_{3} \geq 0$ if and only if $\pi_{3} \leq 2\left(\pi_{1}+\pi_{2}\right)$. Hence, if $\pi_{3}>2\left(\pi_{1}+\pi_{2}\right)$ the nucleolus always leads to the equal division, independently of $p_{3} \leq \pi_{1}+\pi_{2}$.

The average of core's vertices differs from the Shapley value except in the particular case where there is only one strong patent owner. As a "core center" solution concept, it has not been axiomatized and it suffers from not being continuous. It is however interesting to notice that it is given by a simple formula that defines licensing fees that are independent of the number of strong patent owners. We have indeed seen that it imposes weak patent owners and external users to concede half of their profit whatever is $m \geq 1$.

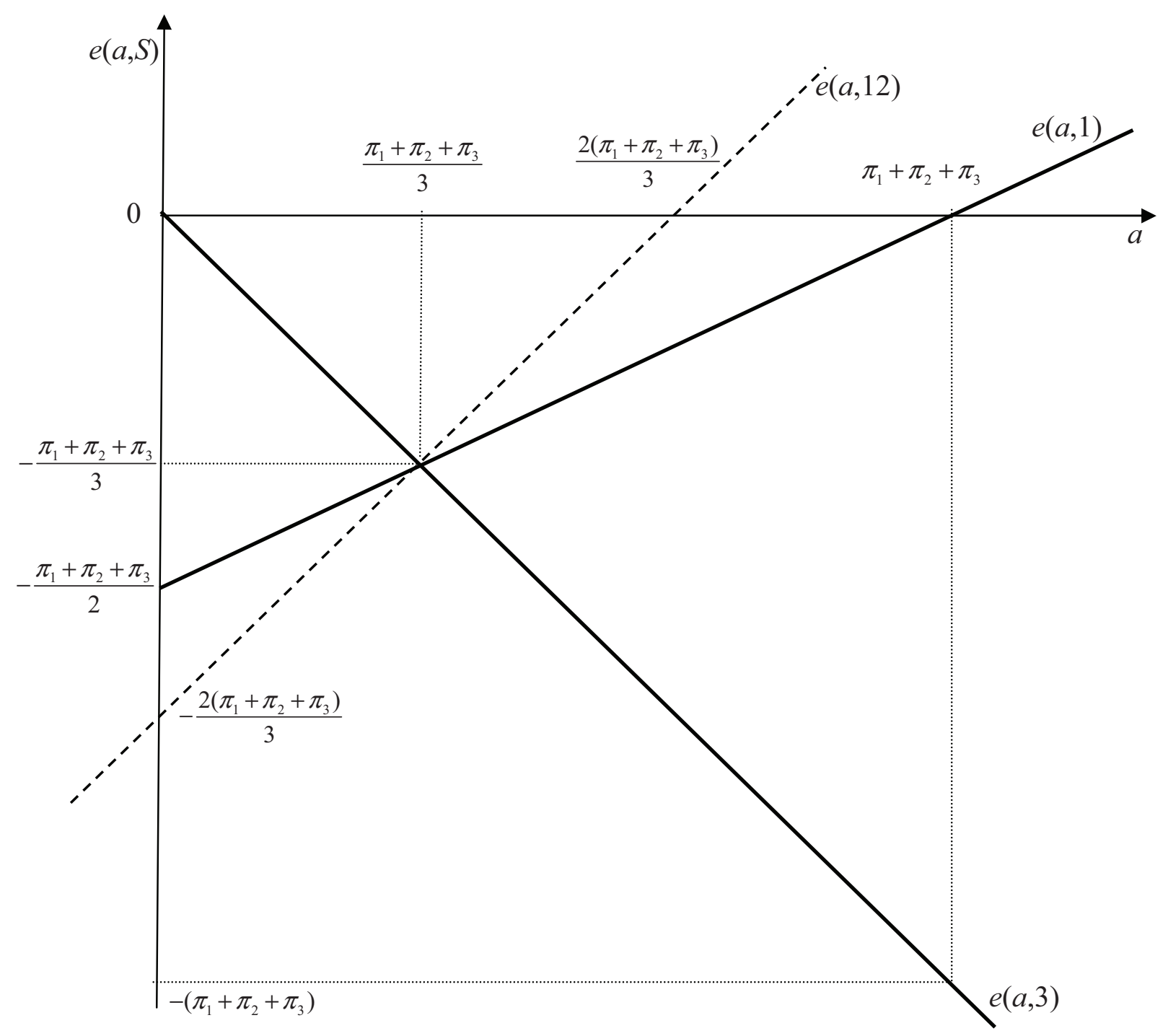

Figure 2 


\section{Concluding remarks}

In order to use cooperative games with side payments, it is necessary to know the worth of all coalitions, whatever is the solution concept that is being considered. Applied to patent games, it means that firms have reached an agreement on the level of net sales that any coalition could raise by selling the standard-based product. Assuming that markets are segmented only slightly simplifies that requirement. There must also be an agreement on which patents are strong and on the value of the other patents. In view of the recommendations of the Shapley value, identifying the strong patents is the most crucial part: being able to convince your partners that a patent you own has no close substitute makes indeed a huge difference. The question of strong patents versus weak patents is discussed by Farell and Shapiro (2008). Specifying the whole range of possible options requires a considerable amount of information. However, according to Layne-Farrar and Lerner (2008) "since modern patent pools tend to grow out of standards, and standards can take years of active debate to define and formalize, members of a patent pool are likely to be quite familiar with the technologies involved and the various standard setting organization members' contributions."

What can we said in general when markets are not segmented? First of all, the simple characterization of the core given in Proposition 1 is lost. Computing numerically the Shapley value is easy once we know the profit that a coalition containing the strong patents owners can secure itself. However, no general formula can be obtained.

Assuming that $\pi(S)$ is known for all $S \supset M$, the patent game is again defined by

$$
\begin{aligned}
v(S) & =\pi(S)-p(N \backslash S) & & \text { if } M \subset S \\
& =0 & & \text { if } M \not \subset S
\end{aligned}
$$

where this time we do not impose additivity of the set function $\pi$. It is quite natural to assume the profit function $\pi$ to be superadditive: merging coalitions can only be beneficial. Convexity is however not granted and therefore the Shapley value may not belong to the core.

It turns out that the licensing fees paid to the weak patent owners remain the same. Indeed, the Shapley value is the sum of the value associated to the game

$$
\begin{array}{rlrl}
w(S) & =\pi(S) & \text { if } M \subset S \\
& =0 & & \text { if } M \not \subset S
\end{array}
$$

and the value associated to the patent game for which $\pi_{i}=0$ for all $i \in N$. 
Referring to the proof of Proposition 2 and using (7), we obtain:

$$
\begin{aligned}
& \text { for } i \notin M: S V_{i}(N, \pi, p)=\sum_{\substack{S \supset M \\
S \ni i}} \alpha_{n}(s)(\pi(S)-\pi(S \backslash i))+\frac{1}{m+1} p_{i} \\
& \text { for } i \in M: S V_{i}(N, \pi, p)=\sum_{\substack{S \supset M \\
S \ni i}} \alpha_{n}(s) \pi(S)-\frac{1}{m(m+1)} \sum_{j \notin M} p_{j}
\end{aligned}
$$

It remains that the strong patent owners are substitutable and, as a consequence, they get an equal amount. The second part of (9) defines the licensing fees paid by the strong patent owners to the weak patent owners. Without more information on the profit function $\pi$, that formula gives no more clues about the licensing fees paid to the strong patent owners.

One last point. In some cases it may be justified to treat players asymmetrically because for instance they differ in size as measured by their market share or production volume. Such situations can be accommodated by using the asymmetric version of the Shapley value, referred to as the weighted Shapley value, for which exogenous weights are assigned to the players. $^{9}$

\section{References}

Baumol, W. and D. Swanson (2005), "Reasonable And Non Discriminatory (RAND) royalties, standard selection and control of market power", Antitrust Law Journal, 73, 1-58.

Cournot, A. (1838), Recherches sur les principes mathématiques de la théorie des richesses, Paris (translated by The Macmillan Company, 1897).

Dehez, P. and D. Tellone (2013), "Data games: Sharing public goods with exclusion", Journal of Public Economic Theory (forthcoming).

Farrell J. and C. Shapiro (2008), "How strong are weak patents?", American Economic Review 98, 1347-1369.

Gillies, D.B. (1953), "Some theorems on n-person games", PhD. Thesis, Princeton University.

Heller, M.A. and R.S. Eisenberg (1998), "Can patents deter innovations? The anticommons in biomedical research, Science 280, 698-701.

Kamien, M.I. and Y. Tauman (1986), "Fees versus royalties and the private value of a patent", Quarterly Journal of Economics 101, 471-492.

\footnotetext{
${ }^{9}$ The asymmetric version of the value was introduced by Shapley in 1953. See Dehez and Tellone (2013) for an application in a data sharing context.
} 
Layne-Farrar A. and J. Lerner (2008), "To join or not to join: examining patent pool participation and rent sharing rules", Social Science Research Network Working Paper.

Layne-Farrar, A., J. Padilla and R. Schmalensee (2007), "Pricing patents for licensing in standard setting organizations: Making sense of FRAND commitments", Antitrust Law Journal, 74, 671-706.

Lerner, J., J. Tirole and M. Strojwas (2003), "Cooperative marketing agreements between competitors: Evidence from patent pools", NBER Working Paper no. 9680, National Bureau of Economic Research.

Maschler, M., Peleg, B., and Shapley, L.S. (1979), "Geometric properties of the kernel, nucleolus and related solution concepts", Mathematics of Operations Research, 4, 303-338.

Muto, S., J. Potters and S. Tijs (1989), "Information market games", International Journal of Game Theory, 18(2), 209-226.

Potters, J. and S. Tijs (1990), "Information market games with more than one informed player", Methods of Operations Research, 63, 313-324.

Shapiro, C. (2001), "Navigating the patent thicket: Cross licenses, patent pools, and standardsetting", in Jaffe A.B., J. Lerner and S. Stern (Eds.), Innovation policy and the economy, vol. 1, NBER, The MIT Press, Cambridge, 119-150.

Shapley, L.S. (1953), "A value for n-person games", in H. Kuhn, and A.W. Tucker, (Eds.), Contributions to the Theory of Games II, Annals of Mathematics Studies 24, Princeton University Press, Princeton, 307-317.

Shapley, L. S. (1971), "Cores of convex games", International Journal of Game Theory 1, 11-26.

Shubik, M. (1982), Game theory for the social sciences, The MIT Press, Cambridge.

Schmeidler, D. (1969), "The nucleolus of a characteristic function game", SIAM Journal of Applied Mathematics 17, 1163-1170.

Young, P.Y. (1985), "Monotonic solutions of cooperative games", International Journal of Game Theory 14, 65-72. 


\section{Documents de travail du BETA}

2013-01 Are Trade Marks and Patents Complementary or Substitute Protections for Innovation Patrick LLERENA, Valentine MILLOT, janvier 2013.

2013-02 Choix stratégique dans l'industrie des plates-formes de loisirs électroniques : une application des modèles 'History-friendly Thierry BURGER-HELMCHEN, février 2013.

2013-03 The Shapley value as a guide to FRAND licensing agreements Pierre DEHEZ, Sophie POUKENS, mars 2013.

La présente liste ne comprend que les Documents de Travail publiés à partir du $1^{\text {er }}$ janvier 2013. La liste complète peut être donnée sur demande.

This list contains the Working Papers written after January 2012, 1rst. The complet list is available upon request. 